\title{
Le paradoxe du risque relatif
}

\author{
F. Bucella \\ Université Libre de Bruxelles, Belgique
}

\begin{abstract}
Résumé. Le paradoxe du risque relatif s'exprime de manière simple. Il consiste à cacher le risque réel, soit le risque absolu, en se concentrant sur l'augmentation ou la diminution du risque, soit le risque relatif. Il y a deux versions du paradoxe. La première postule que l'augmentation du risque peut être importante, mais le risque lui-même reste très faible. Si le risque de base est de 1 sur 10 millions $(0.00001 \%)$, une augmentation de $100 \%$ sera perçue comme très grande, alors que le risque n'est que de 2 sur 10 millions $(0.00002 \%)$. Il s'agit de la version molle du paradoxe du risque relatif, la plus pernicieuse. La deuxième version du paradoxe du risque relatif, la version dure, est la suivante. En se concentrant sur l'augmentation (ou la diminution) du risque, on fait comme si le comportement de base n'avait aucun risque (ou était certain). Si vous dites que les personnes qui consomment un verre de vin par jour augmentent le risque de développer certaines pathologies de $0.5 \%$, vous pourriez croire que les personnes qui ne consomment aucun verre de vin sont protégées. Inversement, si vous dites que consommer des fruits et du vin rouge diminue la probabilité d'apparition de la dysfonction érectile de $19 \%$, vous pourriez croire que les personnes qui ne suivent pas ce régime sont certaines d'être atteintes de la pathologie. Dans un monde idéal, il faudrait exprimer les risques de manière absolue et non relative afin de donner une meilleure perception de la réalité. Cela éviterait les écueils des versions molles et dures du paradoxe du risque relatif.
\end{abstract}

\begin{abstract}
The relative risk paradox is expressed in a simple way. It is to hide the real risk, absolute risk, focusing on increasing or decreasing risk, or relative risk. There are two versions of the paradox. The first postulates that the increase in risk may be important, but the risk itself remains very low. If the base risk is 1 in 10 million $(0.00001 \%$ ), a $100 \%$ increase will be perceived as very large, while the risk is only 2 in 10 million $(0.00002 \%)$. This is the soft version of the relative risk paradox, the most pernicious. The second version of the relative risk paradox, the hard version, is the following. By focusing on increasing (or decreasing) the risk, one acts as if the basic behaviour had no risk (or was certain). If you say that people who consume a glass of wine a day increase the risk of developing certain pathologies by $0.5 \%$, you might think that people who consume no glass of wine are protected. Conversely, if you say that eating fruit and red wine decreases the likelihood of developing erectile dysfunction by $19 \%$, you might think that people who do not follow this diet are sure to have the pathology. In an ideal world, the risks should be expressed in an absolute and not relative way in order to give a better perception of reality. This would avoid the pitfalls of the soft and hard versions of the relative risk paradox.
\end{abstract}

\section{Introduction}

Les études médicales abordent régulièrement la question des risques, par exemple de développer une pathologie. Ceux-ci sont associés à des comportements ou des situations. Quand ils sont évoqués de manière absolue, les risques expriment le pourcentage de la population affecté par le comportement en question (par exemple : consommer quotidiennement un verre de vin rouge). Parfois, les auteurs des études suggèrent une norme comportementale (par exemple : abstinence dans la consommation). Afin de donner une plus grande résonance sur l'impact d'une déviation comportementale par rapport à la norme, les risques sont alors exprimés de manière relative. Lorsqu'ils sont pris en compte dans l'étude même, cela ne prête pas à grande confusion, le lecteur ayant l'ensemble de l'information. Le souci survient lorsque ces risques sont extraits des études. Cités sans précision du contexte, ils donnent une impression faussée des options offertes. La population reçoit une information biaisée sur les différents comportements et les risques qui y sont associés. Cela conduit à des paradoxes.

Le plus curieux des paradoxes est celui du risque relatif. Il consiste à cacher le risque réel, soit le risque absolu, en se concentrant sur l'augmentation ou la diminution du risque, soit le risque relatif. Il y a deux versions de ce paradoxe, qui sont autant de biais. La première version dite molle consiste à mélanger risque relatif et risque absolu. Il s'agit du biais le plus insidieux. L'augmentation du risque peut être très importante, mais le risque lui-même est en réalité très faible. La puissance psychologique du biais est très forte. C'est l'astuce en vigueur lors des soldes. Parler d'une réduction de $80 \%$ n'a pas de sens, si on ne connaît pas le prix. Ce qui intéresse (ou devrait intéresser) l'acheteur est le coût final du bien. Une céramique à 100000 euros, même avec une 《 réduction exceptionnelle de $80 \%$ », reste très onéreuse. Pourtant, qui n'a jamais cédé à se procurer un bien superflu ou dépassant le budget initialement fixé parce que la réduction « en vaut vraiment la peine»? La deuxième 
version du paradoxe du risque relatif est la version dure. En se concentrant sur l'augmentation (ou la diminution) du risque, on fait comme si le comportement de base n'avait aucun risque (ou était certain). Elle ne s'applique pas dans tous les cas. Parfois, ce biais est évité grâce à une connaissance du contexte. L'idéal serait de présenter les résultats des études médicales en risques absolus. Le désavantage est que ceux-ci feront moins souvent la une des journaux. La présente communication vise à (i) rappeler la différence entre risque absolu et risque relatif et à poser le paradoxe du risque relatif dans ses versions molles et dures ; (ii) discuter, dans le cadre du paradoxe $\mathrm{du}$ risque relatif, deux études récentes ayant fait l'objet d'une attention médiatique certaine où la consommation de vin était présentée dans un cas comme augmentant le risque de développer une pathologie, dans l'autre comme le diminuant et (iii) tirer quelques conclusions.

Une partie des résultats exposés ci-après a fait l'objet d'une publication par l'auteur dans la Revue des OEnologues et des techniques vinicoles, dans l'ouvrage « Pourquoi boit-on du vin ? Une enquête insolite et palpitante du Prof. Fabrizio Bucella»ainsi que d'une communication plus grand public reprise notamment en Belgique et en France [1-3]. Celles-ci ne reprenaient pas le concept unifié du paradoxe du risque relatif qui fait l'objet du présent article.

\section{2. Étude du Lancet}

Une étude, publiée le 24 août 2018 par la réputée revue scientifique The Lancet, indiquait qu'une consommation, même modérée, de la molécule d'éthanol (présente dans les boissons alcoolisées) serait mauvaise pour la santé. Réalisée dans 195 pays et s'appuyant sur des données récoltées de 1990 à 2016, l'étude recommandait l'abstinence et la mise en place de mesures politiques sévères pour réduire la consommation :

\begin{abstract}
Nos résultats soulignent la nécessité de revoir les politiques de contrôle de l'alcoolisme et les programmes en faveur de l'abstinence (...) Les gouvernements devraient examiner comment ces recommandations peuvent être mises en oeuvre au sein de leur contexte local (...) en ce compris les accise/taxes sur l'alcool, le contrôle de la disponibilité physique et des heures de vente, et le contrôle de la publicité.
\end{abstract}

L'étude du Lancet est en réalité une méta-étude réalisée par l'Institute for Health Metrics and Evaluation de l'Université de Washington. L'estimation du risque est réalisée avec 3992 données émanant de 592 études. Les éléments sur l'exposition à l'alcool sont au nombre 121029 provenant de 694 sources [4].

L'impact médiatique fut très important. Le sujet fit notamment l'ouverture du journal télévisé de France 2. L'auteur de ces lignes se rappelle être tombé de son fauteuil. Se pourrait-il qu'on ait tout faux ? Que la consommation d'alcool soit vraiment néfaste?

Le Quotidien du Médecin était très clair sur les risques encourus, dès le premier verre :

Il n'y a aucun niveau de consommation d'alcool sûr, et seul zéro gramme d'éthanol par semaine est protecteur, démontre une étude publiée ce 24 août dans «The Lancet», sur la charge mondiale de morbidité liée à l'alcool, d'après les chiffres 2016 de la Global Burden of Disease Study.

À l'aide d'une nouvelle méthodologie, l'étude, qui se veut la plus exhaustive à ce jour sur le fardeau que représente l'alcool, dresse un panorama de sa consommation dans 195 pays.

(...)

L'étude va à l'encontre de l'idée (étayée par des précédentes études, jugées incomplètes) que de faibles quantités d'alcool auraient un effet protecteur. Les bénéfices cardiovasculaires, ou en termes de diabète, chez les femmes sont en effet annihilés par l'importance des risques de cancers, qui augmentent mécaniquement avec la consommation, mais aussi les risques de blessures et de maladies transmissibles.

Seulement zéro verre d'alcool diminue tous les risques sanitaires.

(...)

Les auteurs vont plus loin et appellent à un renouveau des politiques publiques, qui devraient intégrer des recommandations en faveur de l'abstinence, une politique de prix (taxes proportionnelles au contenu en grammes d'alcool et définition d'un prix minimum de vente par unité), le contrôle de l'accès physique aux boissons, et une régulation de la publicité. Les politiques publiques des pays développés, où la consommation est élevée, se doivent d'être plus strictes, tandis que celles des pays à bas ou moyens revenus doivent viser le maintien d'un bas niveau de consommation, lit-on [5].

Grosso modo, ce furent les termes de la dépêche de l'Agence France Presse (AFP), reprise ensuite par l'ensemble des médias, quotidiens, hebdomadaires, des presses radiophonique et télévisuelle.

Quelques mois plus tard, suite à des propos jugés polémiques du ministre français de l'Agriculture ${ }^{1}$, les Décodeurs du journal Le Monde, qui se posent en garant de la vérification des affirmations à l'épreuve des faits, enfonçaient le bouchon si l'on peut dire :

Une analyse, portant sur quelque 700 études sur le sujet et publiée en août 2018 par The Lancet, avait abouti à une conclusion on ne peut plus claire : pour être en bonne santé, il ne faut pas boire.

Aucune quantité ni aucun alcool ne font exception. Les chercheurs ont ainsi battu en brèche le mythe, bien ancré, selon lequel un ou deux

\footnotetext{
${ }^{1}$ Invité de BFM-TV et RMC mercredi 16 janvier 2019, le ministre de l'agriculture et de l'alimentation, Didier Guillaume, était interrogé sur la question de l'alcool en France. Pour lui, si «l'addiction à l'alcool est dramatique », il faut, cependant, distinguer le vin, « qui n'est pas un alcool comme un autre », notamment car il n'est pas celui consommé par les jeunes en boîte de nuit. Ce qu'il a dit : « Le vin n'est pas un alcool comme unautre. (...) L'addiction à l'alcool est dramatique, et notamment dans la jeunesse, avec le phénomène du "binge drinking". Mais je n'ai jamais vu un jeune qui sort de boîte de nuit et qui est saoul parce qu'il a bu du côtes-durhône, du crozes-hermitage, du bordeaux, jamais. Ils boivent des mélanges, de l'alcool fort. » (Les Décodeurs, Le Monde, 16 janvier 2019)
} 
verres de vin quotidiens pourraient avoir des effets bénéfiques [6].

En vérité, ce qu'a affirmé le Lancet n'était pas précisément cela : « aucun niveau de consommation d'alcool n'améliore la santé », mais la subtile nuance a échappé au plus grand nombre [7]. Si on accepte l'affirmation « aucun niveau de consommation d'alcool n'améliore la santé» (certains professionnels mettent en doute l'affirmation en tant que telle), cela ne signifie pas que « pour être en bonne santé, il ne faut pas boire », ainsi que l'affirme le journal Le Monde. On peut fort bien être en bonne santé tout en buvant, c'est d'ailleurs le cas d'une écrasante majorité de la population qui apprécie de temps en temps un verre de vin ${ }^{2}$.

La réception médiatique de l'étude et la confusion qui en découle furent en partie alimentées par le paradoxe $\mathrm{du}$ risque relatif. Le public a compris les éléments suivants. (i) Les personnes abstinentes n'ont pas de risque de développer une maladie liée à l'éthanol. (ii) L'augmentation du risque est forte pour les personnes qui boivent deux verres par jour ( 7 personnes sur 100 développent une pathologie liée à l'éthanol à horizon d'un an). (iii) Pour la dernière catégorie (cinq verres par jour), il existe plus d'une chance sur trois $(37 \%)$ de développer une maladie liée à l'éthanol.

Ces interprétations sont fausses, comme nous le montrons plus loin. Elles découlent toutes du paradoxe $d u$ risque relatif. Dans sa version molle - la plus pernicieuse, le paradoxe du risque relatif confond les risques absolus et relatifs. Les affirmations reprises supra (ii) et (iii) ressortissent à cette catégorie. On cache le risque réel (absolu) derrière une augmentation (ou diminution) de celui-ci (risque relatif), de la même manière que, lors des soldes, on cache le prix d'un bien derrière la réduction affichée. Dans sa version dure, le paradoxe du risque relatif sous-entend un risque zéro pour le comportement de référence. Dans notre cas, on pourrait croire que le risque de développer une pathologie associée à une vie abstinente est zéro ( « pour être en bonne santé, il ne faut pas boire $\gg)$, or ce n'est pas le cas. L'affirmation supra (i) est l'expression de la version dure du paradoxe du risque relatif. Nous explorons ces notions dans les lignes qui suivent.

\section{Paradoxe du risque relatif}

Rappellons la présentation de l'étude du Lancet. Le risque de développer une des 23 boit. .

pathologies liées à l'éthanol à horizon d'un an, si on

- un verre par jour, augmente de $0.5 \%$

- deux verres par jour, augmente de $7 \%$

- cinq verres par jour, augmente de $37 \%$

\footnotetext{
2 Il s 'agit d'une confusion des termes « bon 》 (bonne santé) et 《meilleur 》 (améliore la santé). Sous ce caveat, remarquons que la contraposée logique est appliquée correctement :
}

(alcool $\rightarrow$ meilleure santé) $\leftrightarrow$ (meilleure santé $\rightarrow$ alcool)

En texte : consommer l'alcool n'améliore pas la santé est équivalent à une amélioration de la santé implique une nonconsommation d'alcool (précepte que tous les hôpitaux du monde mettent en application).
Comme nous l'avons évoqué, la présentation de l'étude a mis en oeuvre le paradoxe $d u$ risque relatif. Dans sa version molle, il consiste à confondre le risque réel (ou absolu) et l'augmentation de celui-ci (risque relatif). Expliquons simplement la différence entre risque absolu et risque relatif. Imaginons qu' on vous dise, qu'en traversant la rue devant chez vous, la nuit, vous avez $100 \%$ de chances en plus de vous faire renverser par une voiture. L'écrasante majorité des personnes ne prendra pas le risque de bouger. Pourtant, il ne s'agit pas de $100 \%$ de chances de se faire écraser, ce qui serait une certitude, mais bien de $100 \%$ en plus. En plus par rapport à quoi ? Ce $100 \%$ est un risque relatif (ou augmentation de risque) qui ne permet pas de prendre la bonne décision (traverser ou non la rue). Pour cela, il nous faut connaître la probabilité de se faire écraser en traversant la rue, c'est le risque absolu.

Le paradoxe du risque relatif dans sa version dure se déduit facilement. Si vous traversez la rue en plein jour, vous avez zéro pour cent de chance de vous faire écraser. En vérité, ce n'est pas vrai. Vous avez zéro chance de plus de vous faire écraser par rapport à quelqu'un qui traverse la rue... en plein jour. Normal me direz-vous. Vous regardez l'augmentation du risque par rapport au même comportement (ici traverser la rue en pleine journée). Exprimé de cette façon, le paradoxe du risque relatif disparaît.

Imaginons qu'on précise que la probabilité de ce type d'accident mortel est de $0.00001 \%$. Prendriez-vous le risque de traverser la rue ? Assurément oui. La nuit le risque augmente de $100 \%$, il devient donc 2 sur 10 millions, soit le double du risque en pleine journée. Un risque toujours très faible, soit $0.00002 \%$. Prenez-vous le risque de traverser ? Encore oui.

Il y a bien $100 \%$ de risque en plus de se faire écraser la nuit qu'en plein jour, c'est le risque relatif ou l'augmentation du risque. Cependant, le risque réel de se faire écraser reste extrêmement faible, soit $0.00002 \%$. Il s'agit du risque absolu. La version molle du paradoxe du risque relatif consiste à confondre le risque de se faire renverser par une voiture la nuit $(0.00002 \%$ ou risque absolu) avec l'augmentation du risque par rapport à la même traversée en plein jour (100\% ou risque relatif).

On voit donc, qu'en matière de santé notamment, le risque intéressant à prendre en compte est le risque absolu et non pas l'augmentation du risque ou risque relatif. Dans ce cas, le paradoxe du risque relatif disparaît, tant dans sa version molle que sa version dure. Il ne reste que les risques réels ou absolus, comme si dans le cas des soldes, on n'affichait plus les diminutions des prix, mais simplement le prix final.

\section{Résolution du paradoxe du risque relatif}

Il n'est pas possible de lever le paradoxe du risque relatif, sans calculer les vrais risques, soit les risques absolus. Ce travail a été accompli par le statisticien David Spiegelhalte de l'université de Cambridge. Il a interrogé le Lancet afin d'obtenir les risques absolus, non présentés dans l'étude largement diffusée [8]. On trouve alors les indications suivantes (les risques relatifs ou augmentation des risques 
sont entre parenthèses ; ces risques ont fait les grands titres de la presse, suivant en cela le paradoxe du risque relatif) :

- sur 100000 personnes abstinentes, 914 développent une maladie (liée à l'alcool) à horizon d'un an, risque absolu $0.914 \%$

- sur 100000 personnes qui boivent un verre par jour, 918 développent une maladie, risque absolu de $0.918 \%$ (augmentation de 4 personnes sur 914, soit $0.5 \%$ de risque relatif)

- sur 100000 personnes qui boivent deux verres par jour, 977 développent une maladie, risque absolu de $0.977 \%$ (augmentation de 59 personnes sur 914, soit $7 \%$ de risque relatif)

- sur 100000 personnes qui boivent cinq verres par jour, 1252 développent une maladie, risque absolu de $1.252 \%$ (augmentation de 338 personnes sur 914, soit $37 \%$ de risque relatif) ${ }^{3}$.

On remarque que (i) les personnes abstinentes ne sont pas protégées, elles peuvent également développer une pathologie liée à l'éthanol, le paradoxe du risque relatif dans sa version dure est levé, l'affirmation «pour être en bonne santé, il ne faut pas boire » est contredite ; (ii) les risques des personnes abstinentes et de celles qui boivent un seul verre par jour sont très proches $(0.914 \%$ et $0.918 \%$ respectivement), le paradoxe du risque relatif est levé tant dans sa version dure pour les abstinents, que molle pour les consommateurs d'un verre par jour, l'affirmation « seul zéro gramme d'éthanol par semaine est protecteur» est soit contredite si on estime les risques comparables, soit fortement redimensionnée; (iii) les risques des personnes qui boivent deux verres par jour et cinq verres par jour sont de $0.977 \%$ et $1.252 \%$, le paradoxe du risque relatif est levé dans sa version molle, l'affirmation « il y a $37 \%$ de chances de contracter une pathologie si on boit cinq verres par jour $»$ est contredite.

Lorsque le paradoxe du risque relatif est levé, on comprend qu'il est faux de croire que les abstinents sont protégés, ils peuvent également développer des pathologies liées à l'éthanol, très exactement avec le risque $0.914 \%$. Dans la même veine, il est faux de croire que les personnes qui boivent deux verres par jour ont $7 \%$ de chance (ce qui serait énorme) de tomber malades. Elles ont exactement $0.977 \%$ de chances de tomber malades. Enfin, comme on l'a vu, il est faux de croire que les personnes qui boivent 5 verres par jour ont $37 \%$ de chances d'être atteintes d'une maladie, ce qui serait une hécatombe. Elles ont exactement $1.252 \%$ de chances de tomber malades.

Les trois interprétations de l'étude du Lancet qui ont été évoquées au début de cet article sont donc fausses, voir supra (i, ii et iii) Elles découlaient toutes du paradoxe du risque relatif. Sur la base de ces données, il appartient à chacun de juger de sa consommation et d'adapter, ou non, son comportement.

Il est à noter un élément qui n'est pas accessoire. L'article publié par le Lancet ne donne pas les risques absolus, mais simplement les risques relatifs. La figure de l'étude qui fut abondamment commentée par les médias, donne le « risque relatif pondéré d'alcool

\footnotetext{
${ }^{3}$ Les données peuvent se comparer aux prix des magasins lors des soldes. Même si la réduction est affichée en caractères de taille très grande, le client reçoit l'information sur le prix initial et final que coûte le bien.
}

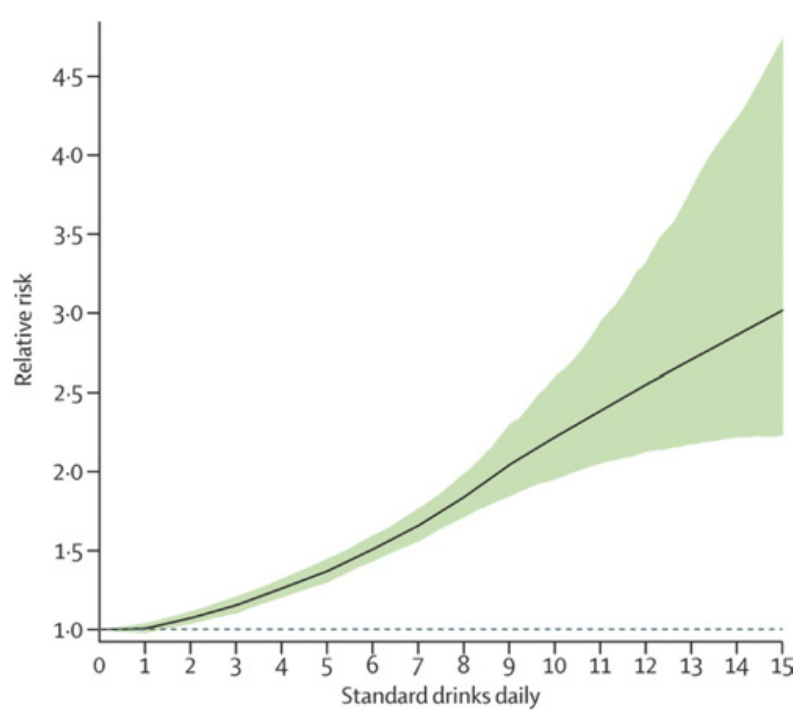

Figure 1. Tirée de l'étude du Lancet. «Risque relatif pondéré d'alcool pour toutes causes imputables, par unités de boisson standard consommées par jour. »

pour toutes causes imputables, par unités de boisson standard consommées par jour $»^{4}$-Fig. 1 à la fin de cet article. Dans le texte de l'article, les auteurs précisent à propos de la figure: « En estimant la courbe de risque relatif pondéré, nous avons constaté que consommer quotidiennement zéro boisson standard [abstinence NDLA] minimisait le risque global de toute maladie $\gg^{5}$. Cette dernière affirmation facilement mais inexactement extrapolée en «les personnes abstinentes sont protégées » est précisément au coeur du paradoxe du risque relatif. L'affaire est étrange car les lignes guides de la revue concernant les méta-études précisent le contraire : « Pour les changements de risque ou les tailles d'effet, donnez des valeurs absolues plutôt que des changements relatifs $»^{6}[9]$.

\section{Modification comportementale}

Pour mieux comprendre le débat entre minimisation du risque et modification comportementale, on peut prendre des exemples de la vie quotidienne. Les habitants de la Commune de Paris (75) sont au nombre de 2200000 (cela fait 22 fois 100 000). Si aucun de ceux-ci ne buvait, on aurait de toute façon 20108 personnes qui développeraient une pathologie liée à l'éthanol. Cela nous rappelle que le risque zéro n'existe pas, soit encore la levée du paradoxe du risque relatif dans sa version dure. Si l'ensemble de ces habitants buvait un verre par jour, il y aurait 20196 personnes qui développeraient une des pathologies. Afin de ne pas être dans les 88 personnes supplémentaires (sur 2200000 ), on peut modifier son comportement et ne pas boire. On court toujours le risque de se trouver dans les

\footnotetext{
${ }^{4}$ Figure 5: Weighted relative risk of alcohol for all attributable causes, by standard drinks consumed per day Age-standardised weights determined by the DALY rate in 2016, for both sexes.

${ }^{5}$ In estimating the weighted relative risk curve, we found that consuming zero standard drinks daily minimised the overall risk of all health loss.

${ }^{6}$ For risk changes or effect sizes, give absolute values rather than relative changes.
} 
20108 personnes qui seront quand même malades tout en ne buvant pas. Le paradoxe du risque relatif est levé dans sa version molle également, la version la plus pernicieuse.

En Belgique, l'Institut de Santé Publique a évalué à 904 le nombre de décès supplémentaires dû aux grosses chaleurs des mois d'été. Rapporté à la taille de la population (11358000), cela donne 8 personnes sur 100000 . Un chiffre comparable aux 4 personnes supplémentaires sur 100000 dont nous avons parlé7. Faut-il éviter les mois d'été et vivre dans un frigo ?

Les humoristes utilisent souvent les paradoxes comme ressort de saynètes. L'art consiste à amener le spectateur dans le coeur du paradoxe sans qu'il s'en rende compte. Je me rappelle d'un comique qui prétendait que prendre l'avion, la voiture ou le vélo est plus dangereux que de ne pas se déplacer. Il en déduisait qu'il fallait rester cloîtré à la maison. Dans la même veine, il remarquait aisément que la vie est une maladie mortelle, dont la probabilité de décès est absolue, soit $100 \%$. Pour protéger les gens de la vie, faut-il les empêcher de vivre ?

\section{Autres biais de l'étude}

Comme nous l'avons vu, la compréhension de l'étude est biaisée en mettant en avant le risque relatif en lieu et place du risque absolu, c'est le paradoxe du risque relatif. Cela semble d'autant plus paradoxal que les lignes guides du Lancet, préconisent d'évoquer les risques en termes absolus et non relatifs.

Les auteurs de l'étude mettent en avant d'autres biais qui sont résumés succinctement ici :

- Estimation de la consommation non-enregistreée ou des productions illégales.

- Les comportements de consommation d'alcool sont considérés constants au cours de l'année.

- Les données des dégâts liés aux véhicules ne sont disponibles qu'aux États-Unis et ont été extrapolées à l'ensemble des autres pays.

- Les données liées aux violences interpersonnelles n'ont pu être récoltées de manière robuste.

- La consommation des personnes âgées de moins de 15 ans n'a pas été prise en compte.

L'association Vin \& Société, soutien inconditionnel de la filière viti-vinicole, fut piquée au vif par la réception de l'étude. Elle a compilé d'autres biais, dont notamment le choix des 23 pathologies retenues, notamment celui de la tuberculose $^{8}$ :

Si le choix de ces problèmes peut être logique pour certains pays, il ne l'est pas partout. Par

\footnotetext{
${ }^{7}$ Sur 100000 personnes qui boivent un verre par jour, 918 développent une maladie, risque absolu de $0.918 \%$ (augmentation de 4 personnes sur 100000 par rapport aux abstinents).

${ }^{8}$ Les chercheurs ont construit un modèle statistique complexe pour déterminer le risque de développer 23 problèmes liés à l'alcool, incluant les maladies cardiovasculaires, sept types de cancer, des maladies non-transmissibles comme la cirrhose du foie et le diabète, des maladies transmissibles comme des infections respiratoires mineures et la tuberculose, la violence et la violence auto-infligée, les blessures liées à la circulation de la route et d'autres blessures non intentionnelles liées à l'empoisonnement ou la noyade.
}

exemple, la prévalence de la tuberculose est devenue faible en Europe de l'Ouest et elle est y peu mortelle. En 2016, ce sont 10.4 millions de nouveaux cas de tuberculose qui sont estimés dans le monde. En France, 4741 nouveaux cas ont été recensés soit $0.05 \%$ des cas dans le monde. Or, toute consommation d'alcool augmente le risque de tuberculose, et l'étude en fait une des principales pathologies liées à l'alcool, ce qui influence ses estimations sur l'impact sanitaire global de l'alcool.

En revanche, les pathologies pour lesquelles la consommation d'alcool peut avoir des bénéfices ne sont pas toutes listées; c'est le cas de la démence et de la mortalitéi globale.

Par ailleurs, alors que beaucoup des 23 pathologies touchent principalement des sujets âgés de plus de 60 ans (AVC, infarctus du myocarde, cancers), l'étude ne pondère pas la mortalitéi par l'âge et les autres causes de mort [10].

C'étaient peu ou prou les conclusions de Walter Willett, professeur en nutrition et épidémiologie à la Harvard T.H. Chan School of Public Health:
S'il n'y a pas de doute sur le fait que la consommation excessive est nocive, il indique que beaucoup de données mettent en évidence un lien entre la consommation modérée et une mortalitéi totale moindre ainsi qu'un risque moindre de maladies cardiaques - ce qui, dit-il, sont des enjeux plus importants pour la plupart des Américains qu'une maladie comme la tuberculose que l'étude du Lancet identifie comme une des principales maladies liées à l'alcool à travers le monde. La tuberculose est très rare aux États- Unis. Les décisions que nous prenons sur la consommation d'alcool aux États-Unis ne devraient pas être influencées par le lien entre alcool et tuberculose [11,12].

\section{Dysfonction érectile}

Dans l'exemple précédent, les risques relatifs donnaient une interprétation anxiogène de la consommation d'éthanol. La levée du paradoxe du risque relatif a permis de cerner les risques réels. Existe-t-il des cas où le risque relatif donne une interprétation trop optimiste d'une consommation de vin ? Une étude de Harvard ${ }^{9}$ publiée le 13 janvier 2016 dans The American Journal of Clinical Nutrition montrait une diminution de la dysfonction érectile pour les consommateurs de fruits et vin rouge [13].

La présentation de l'étude, fut également dithyrambique. Le site de l'université de Harvard précisait :

Les hommes qui consomment seulement trois ou quatre portions hebdomadaires d'aliments et de boissons contenant des flavonoïdes (notamment des baies, des agrumes et du vin rouge) peuvent réduire leur risque de dysfonction érectile. (...) Les chercheurs ont également constaté que les hommes qui étaient physiquement actifs en plus

\footnotetext{
${ }^{9}$ L'étude fut réalisée par la Harvard T.H. Chan School of Public Health et l'université de East Anglia.
} 
de consommer de grandes quantités de flavonoïdes présentaient le risque le plus faible de dysfonction érectile [14].

Un article du journal The Telegraph abondait dans le même sens :

Pourquoi le vin rouge et le cassis sont-ils des superaliments sexuels?

Des scientifiques ont découvert que des aliments riches en flavonö̈des, tels que les myrtilles et le vin rouge, préviennent le dysfonctionnement érectile [15].

Le service public radio-télévisuel belge, la RTBF, reprenait la dépêche AFP :

\begin{abstract}
Plus d'un tiers des hommes ont rapporté souffrir de troubles érectiles, cependant ceux qui avaient une alimentation riche en flavonoïdes étaient moins susceptibles d'en subir. Quelques portions par semaine d'aliments riches de cette molécule faisaient baisser le risque de trouble de $10 \%$. Les meilleurs résultats étaient enregistrés par les hommes de moins de 70 ans. Une alimentation riche en fruits réduisait aussi ce risque de $14 \%$.
\end{abstract}

Pour leur recherche, les scientifiques ont noté les capacités érectiles de plus de 50000 hommes qui avaient une moyenne d'âge de 50 ans en l'an 2000, 2004 et 2008. Ils furent interrogés également sur leurs troubles érectiles passés en remontant jusqu'à 1986. Les chercheurs ont noté des informations concernant leur alimentation tous les quatre ans, en se concentrant sur les sources de flavonoïdes les plus courantes.

La diminution de la dysfonction érectile notée par les chercheurs est de $14 \%$ pour les consommateurs de fruits et de $19 \%$ pour les consommateurs des sources les plus importantes d'anthocyanes, flavones et flavonones : citron, pomme/poire, myrtille, fraise et vin rouge, de préférence à base de pinot noir ${ }^{10}[16]$.

Que signifient exactement ces $19 \%$ ? À nouveau il s'agit d'une diminution de risque ou risque relatif. Revoilà le paradoxe du risque relatif dans sa version molle, celle qui confond les risques absolu et relatif. Afin de trouver les risques absolus, il faut se plonger dans l'étude. Ils sont de $4.04 \%$ pour la population en général et $3.27 \%$ pour les consommateurs réguliers de fruits et de vin rouge. La diminution de 4.04 à 3.27 est bien de $19 \%$.

Dans sa version dure, le paradoxe du risque relatif fait croire que les personnes consommatrices de fruits et vin rouge sont protégées, donc qu'elles ont un risque nul d'être atteintes du syndrome de la dysfonction érectile. Dans ce cas, le contexte permet de lever facilement le paradoxe.

Comme nous l'avions noté dans l'étude du Lancet, l'étude de Harvard T.H. Chan School of Public Health et de l'université de East Anglia ne donne pas les risques absolus mais uniquement les risques relatifs. La figure de cette étude, qui montre ces risques et que je projette à mes étudiants afin de leur montrer comment on peut biaiser la perception, est malicieuse : « Risque de dysfonction érectile associé à la consommation totale de fruits et aux

\footnotetext{
10 La diminution de la dysfonction érectile est $21 \%$ pour les consommateurs de fruits, vin rouge et qui pratiquent une activité physique régulière. Nous notons ce chiffre car il fut abondamment repris par les médias.
}

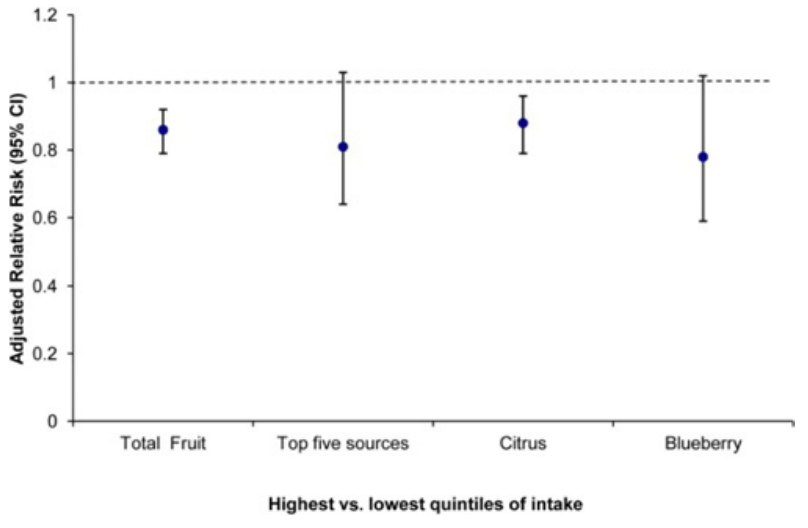

Figure 2. Tirée de l'étude de Harvard. « Risque de dysfonction érectile associé à la consommation totale de fruits et aux 5 sources principales d'anthocyanines, de flavanones et de flavones (fraises, myrtilles, vin rouge, pommes/poires et agrumes) et à la consommation d'agrumes et de myrtilles séparément.»

5 sources principales d'anthocyanines, de flavanones et de flavones (fraises, myrtilles, vin rouge, pommes/poires et agrumes) et à la consommation d'agrumes et de myrtilles séparément $\gg^{11}$-Fig. 2 à la fin de cet article. Il faut se rendre sur le graphique lui-même, lire la légende de l'axe vertical afin de comprendre de quel risque il s'agit : le risque relatif. Une ligne pointillée indique le risque relatif ajusté, celui vis-à-vis duquel se mesurent les chances de diminution de la probabilité d'être atteint de dysfonction érectile, soit l'illustration du paradoxe du risque relatif.

\section{Conclusions}

Nous avons analysé le paradoxe du risque relatif. Celui-ci apparaît en deux versions qui sont autant de biais. $\mathrm{La}$ version molle, la plus courante, cache la véritable étendue du risque concernant une action. Le paradoxe du risque relatif mélange le risque réel avec la diminution ou augmentation de celui-ci (risques absolu et relatif). Comme dans l'exemple des soldes la diminution peut être très importante, mais le prix reste très élevé. Dans l'exemple de l'étude sur les risques encourus relativement à la consommation d'alcool (Lancet), on confond l'augmentation du risque pour les personnes qui boivent deux verres par jour $(7 \%)$ avec le risque réel de développer une pathologie $(0.977 \%)$. Inversement, dans l'exemple sur la protection face à la dysfonction érectile (Harvard), on confond, pour les consommateurs de fruits et vin rouge, la diminution du risque (19\%) avec le risque réel $(3.27 \%)$.

La deuxième version du paradoxe est de cacher le risque du comportement de base, sur lequel est évalué le risque relatif. Il s'agit de la version dure du paradoxe. Celle-ci n'apparaît pas toujours, le contexte permettant parfois de lever la contradiction. Quand apparaît la version dure, elle est pernicieuse. Dans l'étude du Lancet, si vous dites que les personnes qui consomment un verre par jour augmentent le risque de développer une pathologie de $0.5 \%$, vous pourriez croire que les personnes qui ne consomment aucun verre de vin sont protégées ( « pour être

\footnotetext{
11 Risk of erectile dysfunction associated with total fruit intake and intake of the top 5 sources of anthocyanins, flavanones, and flavones (strawberries, blueberries, red wine, apples/pears, and citrus products) and intake of citrus and blueberries separately.
} 
en bonne santé, il ne faut pas boire »). En vérité, il y a lieu de comprendre que les personnes qui ne consomment aucun verre sont aussi sujettes à contracter une des 23 pathologies liées à l'éthanol avec un risque de $0.914 \%$. Le risque pour les abstinents, qui n'est pas négligeable, est passé sous silence.

Dans l'étude de Harvard, on parle de la consommation de fruits et de vin rouge pour diminuer la probabilité d'apparition de la dysfonction érectile (19\%). Vous pourriez croire que les personnes qui ne suivent pas ce régime sont certaines d'être atteintes de la pathologie. Dans ce cas, le contexte permet d'éviter de tomber dans le paradoxe. En vérité, les risques absolus sont, comme nous l'avons vu, de $4.04 \%$ pour la population en général et $3.27 \%$ pour les consommateurs réguliers de fruits et de vin rouge. Le choix de vouloir modifier le régime alimentaire afin de passer de la catégorie de $4.04 \%$ à celle de $3.27 \%$ peut se concevoir, mais il doit être exprimé exactement de cette manière. Évoquer une diminution du risque de $19 \%$ donne une impression faussée de la réalité. On fait croire à un risque différent (version molle du paradoxe du risque relatif), voire que la dysfonction érectile est une issue certaine pour toute personne de plus de 50 ans, ce que le bon sens évacue plutôt rapidement (version dure du paradoxe du risque relatif).

Dans un monde idéal, il faudrait exprimer les risques de manière absolue et non relative afin de donner une meilleure perception de la réalité, notamment dans les études médicales.

La question de notre rapport au vin ne peut se réduire à une diatribe entre esculapes, sur le fait de savoir si la consommation est conseillée médicalement. Dans mon ouvrage « Pourquoi boit- on du vin ? Une enquête insolite et palpitante du Prof. Fabrizio Bucella », on évoque d'autres raisons à notre passion pour ce produit [17]. Le lecteur intéressé y trouvera les développements de cette question.

Athénien. : Est-ce que l'intensité de nos plaisirs et de nos peines, de nos emportements et de nos amours, est surexcitée par l'acte de boire du vin? Clinias : Et grandement même!

Platon, Les Lois.

\section{Références}

[1] Fabrizio Bucella (2018), «Alcool : l'étude du Lancet confond risque relatif et risque absolu », Revue des OEnologues et des techniques vinicoles

[2] Fabrizio Bucella (2019), Pourquoi boit-on du vin? Une enquête insolite et palpitante du Prof. Fabrizio Bucella, Dunod (Paris)

[3] Collectif (2018), « Un scientifique belge d émonte totalement l'étude sur la dangerosité de l'alcool : pas $37 \%$ de risque, mais seulement $1.3 \%$ », RTL. Accessible en ligne : https://www.rtl.be/info/magazine/sante/ un-professeur-belge-demonte-totalement1-etude-sur-la-dangerosite-de-1-alcoolpas-37-de-risque-mais-seulement-13-1058280 . aspx

[4] Collectif (2018), « Alcohol use and burden for 195 countries and territories, 1990-2016: a systematic analysis for the Global Burden of Disease Study $2016 »$, The Lancet. Accessible en ligne : https ://www . thelancet . com/article/S01406736 (18) 31310-2/fulltext

[5] Caroline Garré (2018), « L'alcool néfaste dès les premières gorgées, « The Lancet»appelle à durcir les politiques publiques », Le Quotidien $d u$ Médecin. Accessible en ligne : https : //www. lequotidiendumedecin.fr/ actualites/article/2018/08/24/lalcoolnefaste-des-les-premieres-gorgees-lancetappelle-durcir-les-politiques-publiques 860299

[6] Jonathan Parienté, Samuel Laurent (2019), « Le vin est « un alcool comme un autre », n'en déplaise au ministre de l'agriculture », Les Décodeurs - Le Monde. Accessible en ligne: https://www.lemonde.fr/les-decodeurs/ article/2019/01/16/le-vin-est-un-alcoolcomme-un-autre-n-en-deplaise-au-ministrede-1-agriculture_5409994_4355770.html

[7] Robyn Burton, Nick Sheron (2019), "No level of alcohol consumption improves health ", The Lancet. Accessible en ligne: https://www . thelancet. com/article/S0140-6736 (18)31571$\mathrm{X} /$ fulltext

[8] David Spiegelhalte (2018), "The risk of alcohol (again)", Medium. Accessible en ligne: https:// medium.com/wintoncentre/the-risksof-alcohol-again-2ae8cb006a4a

[9] Collectif (non-daté), "Systematic reviews and metaanalyses in The Lancet: formatting guidelines", The Lancet. Accessible en ligne: http:// www. download. thelancet.com/pb/assets/ raw/Lancet/authors/metaguidelines.pdf

[10] Collectif (2019), « Analyse de l'étude du Lancet », Vin \& Société. Accessible en ligne: http://www.vinetsociete.fr/sites/default/ files/2019-01/Analyse $\% 20$ étude $\% 20$ The $\% 20$ Lancet.pdf

[11] Analyse de l'étude du Lancet. Op. cit

[12] Jamie Ducharme (2018), “A New Study Says Any Amount of Drinking Is Bad for You. Here's What Experts Say", Time. Accessible en ligne: http://time.com/5376552/how-muchalcohol-to-drink-study/

[13] Fabrizio Bucella (2018), L'Antiguide du vin, ce que les autres livres ne vous disent pas, Dunod (Paris)

[14] Collectif (2016), "Flavonoid-rich foods and drinks may prevent erectile dysfunction", Harvard TH Chan - School of Public Health. Accessible en ligne: https : //www.hsph. harvard. edu/news/hsphin-the-news/flavonoid-rich-foods-anddrinks-may-prevent-erectile-dysfunction/

[15] Sarah Knapton (2016), "Why red wine and blackcurrants are sexual superfoods", The Telegraph. Accessible en ligne: https://www . telegraph.co.uk/news/science/sciencenews/12097592/Why-red-wine-and-black currants-are-sexual-superfoods.html

[16] Aed ín Cassidy et al. (2016), "Dietary flavonoid intake and incidence of erectile dysfunction", Ame. J. Clin. Nutri.

[17] Fabrizio Bucella (2019), Pourquoi boit-on du vin ? Une enquête insolite et palpitante du Prof. Fabrizio Bucella. Dunod (Paris) 